\title{
Prevalencia de infraoclusión en molares primarios de niños de 7 y 8 años, Valdivia, Chile, 2019
}

\author{
Prevalence of infraocclusion in primary molars of \\ children aged 7 and 8, Valdivia, Chile, 2019 \\ Prevalência de infraoclusão em molares decíduos de \\ crianças de 7 e 8 anos, Valdivia, Chile, 2019
}

\author{
Francisca Díaz Schiappacasse ${ }^{1}$ ORCID: 0000-0001-6346-1072 \\ Felipe Aguilera-Muñoz ${ }^{1}$ ORCID: 0000-0002-0390-0018 \\ Valentina Cayo-Parra² ORCID: 0000-0003-0842-0408 \\ Katherine Guido-Garay ${ }^{2}$ ORCID: 0000-0003-0686-7943
}

DOI: 10.22592/ode2020n35a2

\section{Resumen}

Objetivo: Determinar la prevalencia de infraoclusión en molares primarios de niños de 7 y 8 años, Valdivia, Chile.

Materiales y métodos: Estudio descriptivo de corte transversal. Se examinaron niños de 7 y 8 años en establecimientos educacionales de Valdivia. Fue evaluada la presencia y severidad de infraoclusión en molares primarios utilizando la clasificación de Brearley \& McKibben. Para establecer diferencias estadísticas entre sexo y presencia de infraoclusión fue realizada la prueba de chi-cuadrado. Además un análisis de ANOVA fue utilizado para establecer diferencias entre la localización de la infraoclusión y el grado de severidad. El nivel de significancia estadística se estableció con un valor de $\mathrm{p}<0,05$.

Resultados: Fueron evaluados 359 niños y un 41,78\% presentó infraoclusión. Según grado de severidad, $82,06 \%$ fueron leves, $15,28 \%$ moderadas y $2,66 \%$ severas. No se encontraron diferencias significativas entre sexo y presencia de infraoclusión. Se evidenciaron diferencias estadísticamente significativas al evaluar localización y grado de severidad $(\mathrm{p}<0,05)$.

Conclusión: Existe una alta prevalencia de infraoclusión en niños de 7 y 8 años en Valdivia, Chile.

Palabras clave: infraoclusión, diente anquilosado, molares primarios, prevalencia.

Instituto de Odontoestomatología, Facultad de Medicina, Universidad Austral de Chile, Valdivia, Chile.

Escuela de Odontología, Universidad Austral de Chile, Valdivia, Chile. 


\section{Abstract}

Objective: To determine the prevalence of infraocclusion in primary molars of children aged 7 and 8 in Valdivia, Chile.

Materials and methods: Descriptive cross-sectional study. Children aged 7 and 8 were examined in educational institutions in Valdivia. The presence and severity of infraocclusion in primary molars was evaluated using the Brearley \& McKibben classification. The chisquare test was performed to establish statistical differences between sex and presence of infraocclusion. In addition, an ANOVA test was used to establish differences between infraocclusion location and degree of severity. The level of statistical significance was established at $\mathrm{p}<0.05$.

Results: Of 359 children evaluated, $41.78 \%$ had infraocclusion. As per degree of severity, $82.06 \%$ of cases were mild, $15.28 \%$ moderate and $2.66 \%$ severe. No significant differences were found between sex and presence of infraocclusion. Statistically significant differences appeared when assessing location and degree of severity $(\mathrm{p}<0.05)$.

Conclusion: There is a high prevalence of infraocclusion in children aged 7 and 8 in Valdivia, Chile.

Keywords: infraocclusion, ankylosed tooth, primary molars, prevalence.

\section{Introducción}

La infraoclusión es una variación del desarrollo que perturba el proceso de erupción de los dientes ${ }^{(1,2)}$ y resulta en un diente que se ubica por debajo del plano oclusal ${ }^{(3-5)}$. La mayoría de los dientes que presentan infraoclusión han entrado en erupción en el plano normal de oclusión y luego han dejado de hacerlo mientras que los dientes adyacentes han continuado,

\section{Resumo}

Objetivo: Determinar a prevalência de infraoclusão em molares decíduos de crianças de 7 e 8 anos, Valdivia, Chile.

Materiais e métodos: Estudo transversal descritivo. Crianças de 7 e 8 anos foram examinadas em estabelecimentos de ensino em Valdivia. A presença e gravidade da infraoclusão em molares decíduos foram avaliadas pela classificação de Brearley \& McKibben.

Para estabelecer diferenças estatísticas entre sexo e presença de infraoclusão, foi realizado o teste do qui-quadrado. Além disso, uma análise ANOVA foi usada para estabelecer diferenças entre a localização da infra-oclusáo e o grau de gravidade. $\mathrm{O}$ nível de significância estatística foi estabelecido com um valor de $\mathrm{p}<0,05$.

Resultados: 359 crianças foram avaliadas e $41,78 \%$ apresentaram infra-oclusão. De acordo com o grau de gravidade, $82,06 \%$ eram leves, $15,28 \%$ moderados e $2,66 \%$ graves. Não foram encontradas diferenças significativas entre sexo e presença de infra-oclusão. Diferenças estatisticamente significantes foram evidenciadas na avaliação da localização e do grau de gravidade $(\mathrm{p}<0,05)$.

Conclusáo: Existe uma alta prevalência de infra-oclusão em crianças de 7 e 8 anos em Valdivia, Chile.

Palavras-chave: infraoclusão, dente anquilosado, molares decíduos, prevalência.

como resultado, el diente afectado permanece en un estado de retención estática, mientras que en las áreas adyacentes, la erupción y el crecimiento alveolar continúan ${ }^{(6)}$.

Esta alteración, también es conocida como erupción incompleta, impactación, diente acortado o sumergido, intrusión, retención secundaria. Sin embargo, los términos correctos y más utilizados son infraoclusión y diente anquilosado ${ }^{(7)}$. La prevalencia en dentición primaria 
es variable, oscila entre $1,3 \%$ al $38,5 \%$ detectado principalmente en niños entre 7 y 8 años de edad, siendo una situación clínica que los odontólogos pediátricos tienen que enfrentar con frecuencia ${ }^{(7-12)}$.

El mecanismo biológico subyacente a la infraoclusión no se conoce bien, se han propuesto muchas teorías para explicar su etiopatogenia en las que se ponen en juego factores genéticos, epigenéticos y ambientales. Probablemente, todos estos factores juegan un papel importante en la determinación de la etiología, la expresión clínica y el grado de severidad de la infraoclusión. La anquilosis localizada entre la raíz y el hueso alveolar podría ser una explicación del fracaso de la erupción antes, durante o después de la erupción completa, pero es importante destacar que, aunque la infraoclusión a menudo se asocia con la anquilosis, la anquilosis no siempre es la causa de la infraoclusión, o sea un diente puede estar infraocluído pero no necesariamente anquilosado. Además, la infraoclusión puede coexistir y estar relacionada con otras anomalías dentales como la hipodoncia, caninos ectópicos e incisivo lateral en forma de clavija ${ }^{(1,13,14)}$.

Estudios previos han reportado que esta alteración afecta principalmente a molares mandibulares primarios ${ }^{(15)}$, sin predisposición por el género y en mayor frecuencia con un grado de severidad leve ${ }^{(9)}$. Al examen clínico, el diente afectado puede presentar falta de movilidad, sonido metálico a la percusión, y se encuentra bajo el plano oclusal. ${ }^{(4,5)}$

Puede generar diversas complicaciones, las cuales se relacionan directamente con el grado de severidad, que pueden incluir: inclinación de dientes adyacentes, pérdida de espacio en el arco dentario, extrusión del proceso dentoalveolar antagonista, mordida abierta lateral, colapso del reborde alveolar, erupción ectópica o la impactación del premolar sucesor ${ }^{(4,16,17)}$ y riesgo de desarrollar lesiones de caries, debido al difícil acceso para una correcta higiene bucal (18). Además, esta alteración se ha relacionado con la presencia de agenesia del sucesor permanente ${ }^{(19,20)}$.

Por lo mencionado anteriormente, el objetivo de este estudio es determinar la prevalencia de infraoclusión en molares primarios de niños de 7 y 8 años en la ciudad de Valdivia, Chile, 2019.

\section{Materiales y métodos}

Se realizó un estudio descriptivo de corte transversal entre los meses de marzo y abril de 2019 en la ciudad de Valdivia, Chile, en el cual se seleccionaron niños de 7 y 8 años de edad, mediante un muestreo aleatorio por conglomerado según establecimiento educacional (público, subvencionados y particulares). De los 29 colegios de la comuna, un total de 17 fueron incluidos en el muestreo para la selección de los niños. Para establecer el tamaño muestral, fueron considerados los datos del CENSO 2017, el cual revela que la población de niños de 7 y 8 años en Valdivia es de 4.258 (Instituto $\mathrm{Na}$ cional de Estadística), una prevalencia esperada de $12,87 \%{ }^{(7)}$. Estableciendo nivel de confianza del $95 \%(Z 1$-alfa $=1,96)$, y un error esperado del $5 \%$, se determinó un tamaño muestral mínimo de 170 niños, la muestra final fue de 359. Este estudio contó con la aprobación del Comité de Ética del Servicio de Salud de Valdivia (Ord n. ${ }^{\circ}$ 520/2019). Los padres y/o tutores firmaron el consentimiento informado respectivo, además de contar con el asentimiento del niño al momento del examen.

Todos los diagnósticos fueron realizados por dos examinadores (V.C y K.G), las cuales se sometieron al proceso de calibración por una experta en el área (F.D), obteniendo un grado de concordancia tanto intra e interobservador superior a 0,8 mediante índice de Kappa.

Todos los exámenes clínicos fueron realizados en los establecimientos educacionales correspondientes, utilizando barreras de protección universal, como lavado clínico de manos, uso de guantes y mascarillas desechables. Cada niño se posicionó frente al investigador, bajo ayuda 
de luz artificial y en máxima apertura bucal, se procedió a realizar el examen clínico utilizando instrumental básico de exploración intraoral y una regla de endodoncia. Durante este, se consideró la presencia de infraoclusión "cuando un diente se encuentra bajo el plano oclusal con respecto a los dientes vecinos, cuando debería haber alcanzado la oclusión" ${ }^{(3-5)}$. Para determinar el "plano oclusal", se utilizó una regla de endodoncia para proyectar una línea desde la cresta marginal mesial del primer molar permanente hasta la punta de la cúspide del canino primario. La posición de la cresta marginal mesial del primer molar permanente, se determinó visualizando donde se encontraba la base de la cresta de la cúspide mesiovestibular como en el estudio de Odeh et al. ${ }^{(13)}$ En los casos en los que había infraoclusión, el grado de severidad se estableció utilizando la clasificación propuesta por Brearley \& McKibben ${ }^{(11)}$ (Tabla $1)$. Se excluyeron de este estudio, molares con destrucción coronaria extensa, obturaciones mesio-ocluso-distal y coronas de acero inoxidable, o condición adicional que no permitieran determinar la altura oclusal, según lo propuesto por Zúñiga-Tertre et al., ${ }^{(9)}$. Además, fueron registrados los datos de sexo, cantidad de molares infraocluídos, molares afectados, grado de severidad (leve, moderada y severa), localización de la infraoclusión (maxilar, mandibular o ambos) y afectación uni o bilateral. La nomenclatura dentaria utilizada en este estudio fue la propuesta por la Fédération Dentaire Internationale (FDI).

En los casos en que hubo duda a la hora de realizar el diagnóstico de infraoclusión o la clasificación del grado de severidad por parte de una de las examinadoras, se tomó una decisión en conjunto.

Todos los datos fueron registrados en una plantilla de google sheets diseñada específicamente para este estudio, la cual fue exportada en valores separados por comas (.csv), para luego realizar su respectivo análisis de datos utilizando el programa estadístico R (v.3.4.1) mediante

\section{Tabla 1. Clasificación de severidad de infraoclusión.}

\begin{tabular}{|l|l|}
\hline Severidad & Definición \\
\hline Leve & $\begin{array}{l}\text { La superficie oclusal se localiza } 1 \mathrm{~mm} \\
\text { aproximadamente por debajo del plano oclusal. }\end{array}$ \\
\hline Moderada & $\begin{array}{l}\text { La superficie oclusal está aproximadamente } \\
\text { a nivel del punto de contacto del diente } \\
\text { adyacente. }\end{array}$ \\
\hline Severa & $\begin{array}{l}\text { La superficie oclusal se localiza por debajo } \\
\text { del punto de contacto o ecuador del diente } \\
\text { adyacente. }\end{array}$ \\
\hline
\end{tabular}

estadística descriptiva. Para establecer diferencias estadísticas entre sexo y presencia de infraoclusión fue realizada la prueba de chi-cuadrado. Además un análisis de ANOVA fue utilizado para establecer diferencias entre la localización de la infraoclusión y el grado de severidad. El nivel de significancia estadística se estableció con un valor de $\mathrm{p}<0,05$.

\section{Resultados}

De los 359 niños examinados, 184 corresponden al sexo femenino (51\%) y 175 al masculino (49\%). Respecto a la presencia de infraoclusión, un $41,78 \%$ de los niños fueron afectados con esta condición, de los cuales $54 \%$ corresponden al sexo femenino y $46 \%$ al masculino (Tabla 2). La distribución en porcentaje de la presencia de infraoclusión por sexo y número de dientes afectados se observan en la Tabla 3. Según el grado de severidad de la infraoclusión, 247 dientes $(82,06 \%)$ presentaron la alteración en grado leve, $46(15,28 \%)$ moderadas y 8

\section{Tabla 2. Distribución de infraoclusión según género.}

\begin{tabular}{|l|c|c|}
\hline Género & N & $\%$ \\
\hline Femenino & 81 & 54 \\
\hline Masculino & 69 & 46 \\
\hline Total & 150 & 100 \\
\hline
\end{tabular}


Tabla 3. Porcentaje de individuos con infraoclusión por sexo y número de dientes afectados.

\begin{tabular}{|l|l|l|l|l|l|l|l|}
\hline $\begin{array}{l}N^{\circ} \text { dientes } \\
\text { Sexo }\end{array}$ & $\mathbf{1}$ & $\mathbf{2}$ & $\mathbf{3}$ & $\mathbf{4}$ & $\mathbf{5}$ & $\mathbf{6}$ & Total \\
\hline Femenino & $40,74 \%$ & $30,86 \%$ & $14,81 \%$ & $12,35 \%$ & $0,00 \%$ & $1,24 \%$ & $100,00 \%$ \\
\hline Masculino & $37,68 \%$ & $43,48 \%$ & $8,70 \%$ & $7,24 \%$ & $0,00 \%$ & $2,90 \%$ & $100,00 \%$ \\
\hline
\end{tabular}

$(2,66 \%)$ severas. La prueba de ANOVA reveló significancia estadística al evaluar este parámetro $(\mathrm{p}<0,05)$. La distribución según los grados de severidad se observan en la Figura 1.

Respecto a la distribución de dientes afectados, el diente con mayor frecuencia de infraoclusión fue el 75 con un $30,56 \%$, seguido por el diente 85 con $29,2 \%$. Los detalles por dientes afectados se observan en la Tabla 4.

Considerando la localización de la infraoclusión, 121 casos $(80,7 \%)$ afectaron la mandíbula, $15(10 \%)$ el maxilar y 14 (9,3\%) ambos (Fig. 2). La prueba de ANOVA reveló significancia estadística al evaluar este parámetro $(\mathrm{p}<0,05)$. Además, 65 (43,3\%) infraoclusiones fueron del tipo unilateral y $85(56,7 \%)$ bilateral.

Al realizar la prueba de chi-2 para establecer diferencias entre la presencia de infraoclusión según sexo, no se encontraron diferencias estadísticamente significativas $(p>0,05)$.

\section{Discusión}

El mecanismo etiológico de la infraoclusión no está claro, se cree que el principal mecanismo podría ser la anquilosis. Existe una teoría que postula que esta alteración sigue un patrón familiar, ya que se encontró esta anomalía en miembros de una misma familia sin diferencias en el género. Kurol y Olson postulan que la infraoclusión es una anomalía hereditaria, que se hereda a través de múltiples factores, ya sea con herencia poligénica o controlada por un solo gen ambientalmente sensible ${ }^{(21,22)}$. Otros autores proponen que pueden haber otros factores involucrados en el mecanismo etiológico de la infraoclusión, como un metabolismo local alterado, trauma e infección local, irritación química o térmica, fallo del crecimiento óseo, presión anormal de la lengua, alteración en la resorción y aposición normal del tejido óseo, enfermedades sistémicas (sífilis congénita, trastornos endocrinos, etc.), posición y dirección anormal del germen y falta de espacio ${ }^{(17)}$

Este estudio contempló el examen clínico visual directo para detectar la presencia de infraoclusión, dado que este método es más apropiado para su diagnóstico ${ }^{(7,8,11)}$. Otra forma de evaluar esta alteración es mediante el examen radiográfico ${ }^{(8,9,13)}$, pero tiene el inconveniente de que es una imagen bidimensional de una estructura tridimensional, lo que ocasiona la sobreposición de estructuras. Esto sumado a la sensibilidad de

Tabla 4. Infraoclusión por diente afectado según severidad.

\begin{tabular}{|c|c|c|c|c|c|c|c|c|c|}
\hline \multirow[t]{2}{*}{ Severidad } & \multicolumn{9}{|c|}{ Diente } \\
\hline & 55 & 54 & 65 & 64 & 75 & 74 & 85 & 84 & Total \\
\hline Leve & 14 & 2 & 13 & 3 & 81 & 33 & 76 & 25 & 247 \\
\hline Moderado & 2 & 1 & 4 & 0 & 7 & 13 & 11 & 8 & 46 \\
\hline Severo & 0 & 0 & 0 & 0 & 4 & 0 & 1 & 3 & 8 \\
\hline Total & 16 & 3 & 17 & 3 & 92 & 46 & 88 & 36 & 301 \\
\hline
\end{tabular}




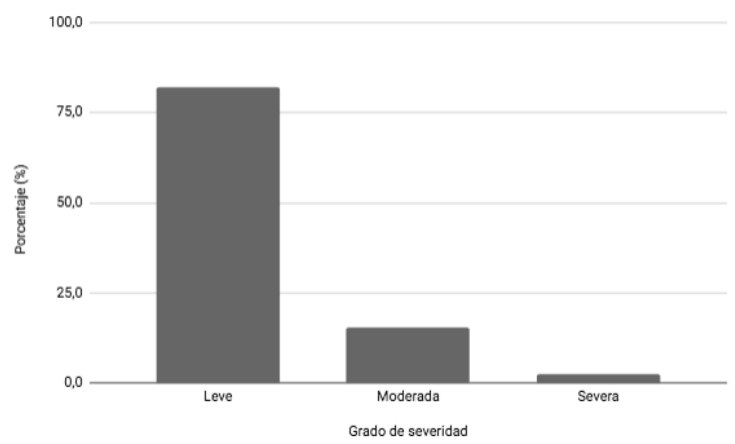

Fig 1. Infraoclusión según grado de severidad. $(\mathbf{p}<0,05)$

la técnica y por consiguiente a la distorsión de imágenes, dificulta la evaluación de severidad de la infraoclusión ${ }^{(23,24)}$.

La prevalencia de infraoclusión de molares primarios obtenida en el presente estudio fue de 41,78\%, demostrando así, que es una alteración frecuente en la población estudiada. No obstante, existen otras investigaciones que difieren de los resultados obtenidos, como el estudio de Kurol (7) que evaluó 1.059 niños suecos, con edades comprendidas entre los 3 y los 12 ańos, registrando una prevalencia general de $8,9 \%$ y de $12,87 \%$ en nińos de 7 y 8 años específicamente. Otros reportes, como el de Zúñiga-Tertre et al., ${ }^{(9)}$ y Cardoso et al., ${ }^{(8)}$, reportaron prevalencias de $10,48 \%$ en niños de 3 a 12 años y 23,1\% en niños de 6 a 9 años respectivamente. La prevalencia obtenida en el estudio de Zúniga-Tertre et al., ${ }^{(9)}$ en el rango etario de 7 y 8 años es de $24,7 \%$. Por el contrario, Steigman et al., ${ }^{(12)}$ que evaluaron a 1.042 niños de 3 a 6 ańos de procedencia israelí, obtuvieron una prevalencia de 38,5\%. Esta variabilidad de resultados, podría ser explicada por diferencias en los criterios diagnósticos y/o metodología utilizada, diferencias étnicas y los rangos etarios considerados $^{(7,8,10,11)}$.

Con respecto a la severidad de la infraoclusión, la de tipo leve fue la más frecuente con un $82,06 \%$, siendo mayor a lo reportado por Brearley \& McKibben ${ }^{(11)}$ y Cardoso et al., ${ }^{(8)}$, en los cuales ambos obtuvieron un 61\%. Los

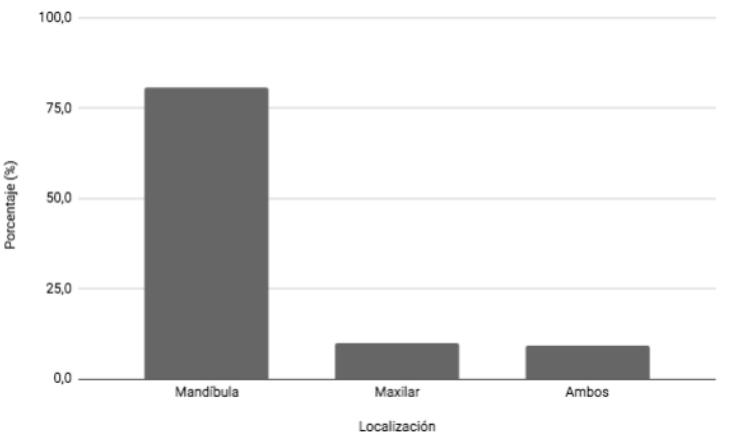

Fig 2. Distribución de Infraoclusión según localización. $(p<0,05)$

casos de infraoclusión severa fueron los menos frecuentes, al igual que en el estudio de Odeh et al., ${ }^{(13)}$, donde sólo se observaron tres casos.

En cuanto al número de dientes infraocluídos por individuo, fue más frecuente observar uno o dos molares primarios afectados, similar a los resultados reportados por Zúñiga-Tertre et al y Brearley \& McKibben. ${ }^{(9,11)}$

Según $\operatorname{Kurol}^{(7)}$, el primer molar mandibular primario es el diente más comúnmente afectado en niños menores de 9 años, después de esta edad, predomina la infraoclusión del segundo molar primario. Zúniga-Tertre et al., ${ }^{(9)}$ reportó resultados similares, encontrando que el molar primario más afectado fue el primer molar mandibular derecho con un 40,1\%, seguido del primer molar mandibular izquierdo $(27,7 \%)$. En nuestro estudio, encontramos que el segundo molar mandibular izquierdo fue el más afectado con un $30,5 \%$, seguido por el segundo molar mandibular derecho (29,2\%).

En la muestra, no existió un patrón determinado de infraoclusión respecto a la aparición en el sector derecho u izquierdo, o la localización del tipo uni o bilateral, al igual que lo reportado por Kurol ${ }^{(7)}$ y Brearley \& McKibben ${ }^{(11)}$.

Existe consenso general de que la infraoclusión se localiza predominantemente en los molares mandibulares ${ }^{(8-13)}$ hasta 10 veces más que en los molares del maxilar según lo registrado por $\mathrm{Ku}$ rol $^{(7)}$, similar a lo encontrado en nuestra muestra. 
Por otra parte, la presencia de infraoclusión fue levemente mayor en las niñas, sin embargo, no hubo diferencias estadísticamente significativas al evaluar la presencia de infraoclusión y sexo, al igual que lo reportado por Zuñiga et al. ${ }^{(9)} \mathrm{y}$ Cardoso et al. ${ }^{(8)}$.

Entre las limitaciones de este estudio, se incluye el reducido rango de edad en comparación con otros reportes ${ }^{(8,9)}$, lo que nos impide establecer si en Chile la población estudiada es la más afectada. Por otra parte, a pesar de que el acuerdo interexaminador logrado en el estudio fue alto, se podría mejorar la precisión en la determinación de la severidad al utilizar modelos de yeso, lo que se sugiere tener en consideración para futuras investigaciones.

En conclusión, la prevalencia de infraoclusión de molares primarios en la población estudiada es alta, siendo una alteración frecuente en la población infantil de 7 y 8 años, presentándose mayoritariamente en grado leve, afectando principalmente molares mandibulares. Se recomienda elaborar medidas interceptivas para el diagnóstico precoz y un adecuado manejo interdisciplinario.

\section{Agradecimientos}

Esta investigación está basada en una tesis realizada por Valentina Cayo y Katherine Guido como requerimiento para la obtención del grado académico de Licenciado en Odontología de la Universidad Austral de Chile, Junio, 2019.

\section{Conflicto de interés}

Los autores declaran no tener conflictos de interés con respecto a la autoría y /o publicación del artículo.

\section{Contribución de autoría}

1. Concepción y diseño del estudio

2. Adquisición de datos

3. Análisis de datos

4. Discusión de los resultados

5. Redacción del manuscrito

6. Aprobación de la versión final del manuscrito

F.D.S ha contribuido en $1,4,5,6$.

F.A.M. ha contribuido en 1, 3, 4, 5, 6 .

V.C.P. ha contribuido en 1, 2, 4, 5, 6.

K.G.G. ha contribuido en 1, 2, 4, 5, 6 .

\section{Referencias}

1. Cobourne MT, Sharpe PT. Diseases of the tooth: the genetic and molecular basis of inherited anomalies affecting the dentition. Wiley Interdiscip. Rev. Dev. Biol. 2013; 2(2): 183-212.

2. Brook AH. Multilevel complex interactions between genetic, epigenetic and environmental factors in the aetiology of anomalies of dental development. Archives of Oral Biology. 2009; 54, Suppl 1, S3-17.

3. Shalish M, Peck S, Wasserstein A, Peck L. Increased occurrence of dental anomalies associated with infraocclusion of deciduous molars. Angle Orthod. 2010;80 (3):440-5.

4. Ekim SL, Hatibovic-Kofman S. A treatment decision-making model for infraoccluded primary molars. Int J Paediatr Dent. 2001;11 (5):340-46.

5. Darling A, Levers B. Submerged human deciduous molars and ankylosis. Archives of Oral Biology. 1973;18 (8):1021-038.

6. Andersson L, Blomlöf L, Lindskog S, Feiglin B, Hammarström L. Tooth ankylosis. Clinical, radiographic and histological assessments. International journal of oral surgery. 1984;13 (6):423-31.

7. Kurol J. Infraocclusion of primary molars: an epidemiologic and familial study. Community Dent. Oral Epidemiol. 1981;9 (2):94-02.

8. Cardoso CS, Maroto ME, Soledad MA, Barbería EL. Primary molar infraocclusion: frequen- 
cy, magnitude, root resorption and premolar agenesis in a Spanish sample. Eur. J. Paediatr. Dent. 2014;15 (3):258-64.

9. Zúñiga M, Lucavechi T, Barbería E. Distribución y gravedad de las infraoclusiones de molares temporales. RCOE. 2004;9 (1):53-9.

10. Koyoumdjisky-Kaye E, Steigman S. Submerging primary molars in Israeli rural children. Community Dent Oral Epidemiol. 1982;10 (4):204-8.

11. Brearley L, McKibben D. Ankylosis of primary molar teeth. I. Prevalence and characteristics. ASDC J. Dent. Child. 1973;40 (1):54-3.

12. Steigman S, Koyoumdjisky-Kaye E, Matrai Y. Submerged deciduous molars in preschool children: an epidemiologic survey. J. Dent. Res. 1973;52 (2):322-26.

13. Odeh R, Mihailidis S, Townsend G, Lähdesmäki R, Hughes T, Brook A. Prevalence of infraocclusion of primary molars determined using a new 2D image analysis methodology. Aust. Dent. J. 2016;61 (2):183-89.

14. Proffit W, Frazier-Bowers S. Mechanism and control of tooth eruption: overview and clinical implications. Orthod. Craniofac. Res. 2009;12 (2):59-66.

15. Henderson H. Ankylosis of primary molars: a clinical, radiographic, and histologic study. ASDC journal of dentistry for children. 1979;46 (2):117-22.
16. Becker A, Karnei-R'em R, Steigman S. The effects of infraocclusion: Part 3. Dental arch length and the midline. Am. J. Orthod. Dentofacial Orthop. 1992;102 (5):427-33.

17. Douglass J, Tinanoff N. The etiology, prevalence, and sequelae of infraclusion of primary molars. ASDC J. Dent. Child. 1991;58 (6):48183.

18. Noble J, Karaiskos N, Wiltshire W. Diagnosis and management of the infraerupted primary molar. Br. Dent. J. 2007;203 (11):632-34.

19. Baccetti, T. A controlled study of associated dental anomalies. Angle Orthod. 1998;68 (3):267-74.

20. Bjerklin K, Kurol J, Valentin J. Ectopic eruption of maxillary first permanent molars and association with other tooth and developmental disturbances. Eur. J. Orthod. 1992;14 (5):36975.

21. Kurol J, Olson L. Ankylosis of primary molars a future periodontal threat to the first permanent molars. Eur. J. Orthod. 1991;13 (5):404-09.

22. Kurol J, Magnusson B. Infraocclusion of primary molars: a histologic study. Scand J Dent Res. 1984;92 (6):564-76.

23. Rubio E, Cueto M, Suárez R, Frieyro J. Técnicas de diagnóstico de la caries dental. Descripción, indicaciones y valoración de su rendimiento. Bol. pediatr. 2006;46 (195): 23-1.

24. García C. Anatomía del error en radiología. Rev. chil. radiol. 2003;9 (3):144-50.

Felipe Aguilera Muñoz: faguileramunoz@gmail.com 